Pacific Journal of Mathematics

AUTOMORPHISMS OF COMPACT KLEIN SURFACES WITH E WIS MAY 


\title{
AUTOMORPHISMS OF COMPACT KLEIN SURFACES WITH BOUNDARY
}

\author{
CoY L. MAY
}

\begin{abstract}
A Hurwitz ramification formula for morphisms of compact Klein surfaces is obtained and used to show that a compact Klein surface of genus $g \geqq 2$ with nonempty boundary cannot have more than $12(g-1)$ automorphisms.
\end{abstract}

o. Introduction. Let $X$ be a compact Klein surface [1], that is, $X$ is a compact surface with boundary together with an equivalence class of dianalytic atlases on $X$. A homeomorphism $f: X \rightarrow X$ of $X$ onto itself that is dianalytic will be called an automorphism of $X$.

A natural task is to seek an upper bound for the order of the automorphism group of $X$ when $X$ is of (algebraic) genus $g \geqq 2$. The corresponding result for Riemann surfaces is well-known; Hurwitz [2] showed that a compact Riemann surface of genus $g \geqq 2$ cannot have more than $84(g-1)$ (orientation preserving) automorphisms. Using this result it is easy to show that the upper bound in the Klein surface case cannot be larger than $84(g-1)$. In fact, Singerman [6] has exhibited a Klein surface without boundary of genus 7 that has $504=84(7-1)$ automorphisms.

In this paper then we concentrate on Klein surfaces with boundary. We obtain a Hurwitz ramification formula for morphisms of Klein surfaces and show that a compact Klein surface with boundary of genus $g \geqq 2$ cannot have more than $12(g-1)$ automorphisms. We also show that the bound $12(g-1)$ is the best possible.

1. Let $X$ be a Klein surface. The boundary of $X$ will be denoted $\partial X$. Let $X^{\circ}=X \backslash \partial X$. $X^{\circ}$ will be called the interior of $X$.

Let $p \in X$. Then let $n_{p}=1$ if $p \in \partial X$ is a boundary point of $X$, and let $n_{p}=2$ if $p \in X^{\circ}$ is an interior point of $X$.

Now we recall the definition of a morphism of Klein surfaces [1, page 17]. Let $\mathscr{C}^{+}=\{z \in \mathscr{C} \mid \operatorname{Im}(z) \geqq 0\}$, and let $\phi: \mathscr{C} \rightarrow \mathscr{C}^{+}$be the folding map, so that $\phi(\alpha+\beta i)=\alpha+|\beta| i$.

Definition. Let $X, Y$ be Klein surfaces and $g: X \rightarrow Y$ a continuous map. Then $g$ is a morphism if $g(\partial X) \subset \partial Y$ and if for every point $p \in X$ there exist dianalytic charts $(U, z)$ and $(V, w)$ at $p$ and $g(p)$ respectively and an analytic function $G$ on $z(U)$ such that the following diagram commutes: 


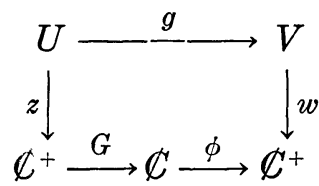

Let $g: X \rightarrow Y$ be a nonconstant morphism of Klein surfaces. Let $x \in X$. We can find dianalytic charts $(U, z)$ and $(V, w)$ at $x$ and $g(x)$ respectively, such that $z(x)=0=w(g(x)), g(U) \subset V$, and such that $\left.g\right|_{U}$ has the form

$$
\left.g\right|_{U}=\left\{\begin{array}{l}
w^{-1} \circ \phi \circ\left( \pm z^{e}\right) \text { if } g(x) \in \partial Y \\
w^{-1} \circ\left( \pm z^{e}\right) \text { if } g(x) \in Y^{\circ}
\end{array}\right.
$$

where $e$ is an integer, $e \geqq 1[1$, pages 27-30]. The integer $e$ is called the ramification index of $g$ at $x$ and will be denoted $e_{g}(x)$. We say that $g$ is ramified at $x$ if $e_{g}(x)>1$; otherwise we say that $g$ is unramified at $x$. Also, the relative degree of $x$ over $g(x)$, denoted $d_{g}(x)$, is defined by

$$
d_{g}(x)=\frac{n_{x}}{n_{g(x)}} .
$$

Note that $d_{g}(x)=2$ if $x \in X^{\circ}$ and $g(x) \in \partial Y$; otherwise $d_{g}(x)=1$.

Definition. A nonconstant morphism $g: X \rightarrow Y$ between two Klein surfaces will be called a ramified r-sheeted covering of $Y$ if for every point $y \in Y$,

$$
\sum_{x \in g^{-1}(y)} e_{g}(x) \cdot d_{g}(x)=r
$$

In fact, every nonconstant morphism between two compact Klein sufaces is a ramified $r$-sheeted covering for some $r$ [1, page 102].

Now let $X, Y$, and $T$ be Klein surfaces, $g: X \rightarrow Y$ and $f: Y \rightarrow T$ be nonconstant morphisms. Then $f \circ g: X \rightarrow T$ is a nonconstant morphisms [1, page 19]. Also, if $g$ is a ramified $r$-sheeted covering of $Y$ and $f$ is a ramified $m$-sheeted covering of $T$, then it is easily seen that $f \circ g$ is a ramified $m r$-sheeted covering of $T$.

Let $X$ be a Klein surface. We will denote the automorphism group of $X$ by $\operatorname{Aut}(X)$. If $X$ is orientable, we will denote the subgroup of orientation preserving automorphisms by $\operatorname{Aut}^{+}(X)$.

TheOREM 1. Let $X$ be a compact Klein surface and let $G \subset \operatorname{Aut}(X)$ be a finite group of automorphisms of $X$. Then the quotient space $\Phi=X / G$ has a unique dianalytic structure such that the canonical map $\pi: X \rightarrow \Phi$ is a morphism of Klein surfaces. Moreover, if $|G|=r$, 
then $\pi$ is a ramified $r$-sheeted covering of $\Phi$.

Proof. Alling and Greenleaf have shown that $\Phi$ has a unique dianalytic structure such that $\pi$ is a morphism [1, pages 52-56]. Actually, in the case of a finite group action (they consider the action of a discontinuous group), their proof shows that $\pi$ is a ramified $r$-sheeted covering of $\Phi$.

2. Let $Y$ be a compact Klein surface, and let $E$ be the field of all meromorphic functions on $Y . E$ is an algebraic function field in one variable over $\boldsymbol{R}$, and as such has an algebraic genus g. We will refer to this nonnegative integer $g$ as the genus of the compact Klein surface $Y$. In case $Y$ is a Riemann surface, $g$ is equal to the topological genus of $Y$. For more details, see [1].

Henceforth the term Klein surface will be reserved for those Klein surfaces $X$ that are not Riemann surfaces, that is, for those $X$ that are nonorientable or have nonempty boundary or both.

Let $X$ be a compact Klein surface. Let $\left(X_{c}, \pi, \sigma\right)$ be the complex double of $X$, that is, $X_{c}$ is a compact Riemann surface, $\pi: X_{c} \rightarrow X$ is an unramified 2-sheeted covering of $X$, and $\sigma$ is the unique antianalytic involution of $X_{c}$ such that $\pi=\pi \circ \sigma$. For more details, see $[1$, pages $37-40]$. It is well-known that the genus of $X$ is equal to the genus of its complex double $X_{c}$. The complex double also has the following important property [1, page 39]:

Proposition 1. Let $M$ be a compact Riemann surface, $X$ a compact Klein surface, and $f: M \rightarrow X$ a nonconstant morphism. Then there exists a unique analytic map $\rho: M \rightarrow X_{c}$ such that $\pi \circ \rho=f$.

We use the complex double to obtain a Hurwitz ramification formula for morphisms of compact Klein surfaces.

Theorem 2. Let $X$ and $Y$ be compact Klein surfaces (that are not Riemann surfaces), and let $f: X \rightarrow Y$ be a ramified r-sheeted covering of $Y$. Let $g$ be the genus of $X, \gamma$ the genus of $Y$. Then

$$
2 g-2=r(2 \gamma-2)+\sum_{x \in X} n_{x}\left(e_{f}(x)-1\right) .
$$

Proof. Let $\left(X_{c}, \pi, \sigma\right)$ and $\left(Y_{c}, \nu, \tau\right)$ denote the complex doubles of $X$ and $Y$ respectively. By Proposition 1, there exists a unique analytic map $\tilde{f}: X_{c} \rightarrow Y_{c}$ such that the following diagram commutes: 


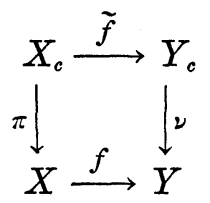

$f \circ \pi=\nu \circ \tilde{f}$ is a ramified $2 r$-sheeted covering of $Y$. But $\tilde{f}$ is a nonconstant analytic mapping between compact Riemann surfaces. Thus $\tilde{f}$ is a ramified $m$-sheeted covering of $Y_{c}$ for some $m$ [3, page 15]. Since $\nu$ is a 2-sheeted covering, clearly $m=r$. Then, since a Klein surface and its complex double have the same genus, the classical Hurwitz ramification formula [3, page 16] gives

$$
(2 g-2)=r(2 \gamma-2)+\sum_{p \in X_{c}}\left(e_{\tilde{f}}(p)-1\right) .
$$

Let $p \in X_{c}$ and note that $e_{\tilde{f}}(p)=e_{f}(\pi(p))$, since $e_{\tilde{f}}^{\tilde{f}}(p)=e_{\nu^{\circ} \tilde{f}}(p)=$ $e_{f^{\circ} \pi}(p)=e_{f}(\pi(p))$.

Therefore

$$
\begin{aligned}
(2 g-2) & =r(2 \gamma-2)+\sum_{p \in X_{c}}\left(e_{f}(\pi(p))-1\right) \\
& =r(2 \gamma-2)+\sum_{x \in X} n_{x}\left(e_{f}(x)-1\right) .
\end{aligned}
$$

Finally, we recall how the automorphism group of a compact Klein surface can be obtained from that of its complex double [1, page 79]:

Proposition 2. Let $X$ be a compact Klein surface with complex double $\left(X_{c}, \pi, \sigma\right)$. Then

$$
\operatorname{Aut}(X) \cong\left\{g \in \operatorname{Aut}^{+}\left(X_{c}\right) \mid \sigma \circ g \circ \sigma=g\right\} .
$$

CoRollary. If $X$ is a compact Klein surface of genus $g \geqq 2$, then

$$
|\operatorname{Aut}(X)| \leqq 84(g-1)
$$

Thus Aut $(X)$ is finite group.

Proof. The genus of $X_{c}$ is $g$, so that the corollary follows immediately from the Proposition and Hurwitz's bound for $\left|\operatorname{Aut}^{+}\left(X_{c}\right)\right|$.

3. Applications. Let $X$ be a compact Klein surface of genus $g$, and let $G \subset \operatorname{Aut}(X)$ be a finite group of automorphisms of $X$ of order $|G|=r$. By Theorem 1 , the quotient space $\Phi=X / G$ is a compact Klein surface and the canonical map $\pi: X \rightarrow \Phi$ is a ramified $r$-sheeted covering of $\Phi$. Let $\gamma$ denote the genus of $\Phi$. 
Let $p \in \Phi$. We will call the set $\pi^{-1}(p)$ the fiber above $p$. If $g \in \operatorname{Aut}(X)$ then $g(\partial X)=\partial X$ and $g\left(X^{\circ}\right)=X^{\circ}$. Therefore either $\pi^{-1}(p) \subset \partial X$ or $\pi^{-1}(p) \subset X^{\circ}$. Equivalently, if $x, y \in X$ such that $\pi(x)=\pi(y)$, then $d_{\pi}(x)=d_{\pi}(y)$.

Let $S_{x}=\{g \in G \mid g(x)=x\}$ be the stabilizer subgroup of $G$ of a point $x \in X$. We can find a dianalytic chart $(U, z)$ at $x$ such that $g(U)=U$ for all $g \in S_{x}$. Let $S_{x}^{\prime}=\left\{g \in S_{x} \mid z \circ g \circ z^{-1}\right.$ is analytic $\}$. Clearly $S_{x}^{\prime}$ is independent of the choice of $(U, z)$. Either $S_{x}=S_{x}^{\prime}$ or $S_{x}^{\prime}$ is a subgroup of index 2. $S_{x}=S_{x}^{\prime}$ in case (i) $x \in X^{\circ}$ and $\pi(x) \in \Phi^{\circ}$ or (ii) $x \in \partial X$ and $e_{\pi}(x)=1$; otherwise $S_{x} \neq S_{x}^{\prime}$. The ramification index $e_{\pi}(x)$ is the order of $S_{x}^{\prime}$ in case $x \in X^{\circ}$ and $\pi(x) \in \partial \Phi$; otherwise $e_{\pi}(x)$ is the order of $S_{x}$. For more details, see [1, page 52-56]. If $\pi(x)=\pi(y)$, then clearly there are isomorphisms $S_{x} \cong S_{y}$ and $S_{x}^{\prime} \cong S_{y}^{\prime}$, so that $e_{\pi}(x)=e_{\pi}(y)$ in any case.

If $\pi$ is ramified at a point $x \in X$ and $\pi(x)=p$, then we will say that $\pi$ is ramified above $p$.

Now the quotient map $\pi: X \rightarrow \Phi$ is ramified above a finite number of points of $\Phi$, say $a_{1}, \cdots, a_{\omega}$. Let $k_{i}$ denote the ramification index $e_{\pi}(x)$ of any point $x$ such that $\pi(x)=a_{i}$. We will write $n_{i}=n_{a_{i}}$.

Fix $a_{i}$. First suppose that if $\pi(x)=a_{i}$, then the relative degree $d_{\pi}(x)=1$, i.e., $n_{x}=n_{a_{i}}=n_{i}$. Then there are $r / k_{i}$ points in the fiber $\pi^{-1}\left(a_{i}\right)$, and

$$
\begin{aligned}
\sum_{x \in \pi-1\left(a_{i}\right)} n_{x}\left(e_{\pi}(x)-1\right) & =\frac{r}{k_{i}} \cdot n_{i} \cdot\left(k_{i}-1\right) \\
& =r n_{i}\left(1-\frac{1}{k_{i}}\right) .
\end{aligned}
$$

Now suppose that if $\pi(x)=a_{i}$, then $d_{\pi}(x)=2$, so that $n_{x}=2$, $n_{i}=1$. In this case there are $r / 2 k_{i}$ points in the fiber $\pi^{-1}\left(a_{i}\right)$, and

$$
\begin{aligned}
\sum_{x \in \pi-1\left(a_{i}\right)} n_{x}\left(e_{\pi}(x)-1\right) & =\frac{r}{2 k_{i}} \cdot 2 \cdot\left(k_{i}-1\right) \\
& =r n_{i}\left(1-\frac{1}{k_{i}}\right) .
\end{aligned}
$$

Therefore the Hurwitz ramification formula (Theorem 2) can be rewritten in the following form:

$$
\frac{2 g-2}{r}=2 \gamma-2+\sum_{i=1}^{\omega} n_{i}\left(1-\frac{1}{k_{i}}\right) \text {. }
$$

Henceforth we assume that $X$ is of genus $g \geqq 2$. Then, by the corollary to Proposition 2, Aut $(X)$ is a finite group, so that in our calculations here we can let $G=\operatorname{Aut}(X)$. The calculations will be divided into several cases. 
A. $\gamma \geqq 1$.

First suppose that $\gamma \geqq 2$. Then, immediately from $\left(^{*}\right)$, we have $(2 g-2) / r \geqq 2$. Thus $r \leqq g-1$.

Now suppose $\gamma=1$. Then $\omega \neq 0$, and

$$
\frac{2 g-2}{r} \geqq n_{1}\left(1-\frac{1}{k_{1}}\right) \geqq 1-\frac{1}{k_{1}} \geqq \frac{1}{2} .
$$

Hence $r \leqq 4(g-1)$.

B. $\gamma=0$, three lemmas.

Recall that there are two compact Klein surfaces of genus zero, the disc $D$ and the real projective plane B. Each has a unique dianalytic structure [1, pages 59-60].

Note that with $\gamma=0,\left(^{*}\right)$ implies that $\omega \geqq 2$.

In the following lemmas we will assume that the Klein surface $X$ has nonempty boundary. Then the quotient space $\Phi$ has nonempty boundary, and since $\gamma=0, \Phi$ is the disc $D$ (with its unique dianalytic structure).

Lemma 1. Suppose $\partial X \neq \varnothing$. If $\pi$ is ramified at a boundary point $x \in \partial X$, then the ramification index $e_{\pi}(x)=2$.

Proof. Let $e=e_{\pi}(x)$. $\pi(x) \in \partial D$, of course.

We can find dianalytic charts $(U, z)$ and $(V, w)$ at $x$ and $\pi(x)$ respectively, such that $z(x)=0=w(\pi(x)), \pi(U) \subset V$, and such that

$$
\left.\pi\right|_{U}=w^{-1} \circ \phi \circ\left( \pm z^{e}\right)
$$

$e \geqq 2$, since $\pi$ is ramified at $x$. Suppose $e>2 . \quad z(U)$ is an open subset of $C^{+}$about the origin. Thus for a small enough real number $t>0$, both the points $\xi_{1}=t, \xi_{2}=t \exp (2 \pi i / e)$ belong to $z(U)$. Then $z^{-1}\left(\xi_{1}\right) \in \partial X$ and $z^{-1}\left(\xi_{2}\right) \in X^{\circ}$, and clearly $\pi\left(z^{-1}\left(\xi_{1}\right)\right)=\pi\left(z^{-1}\left(\xi_{2}\right)\right)$. But for each point $p \in D$, either $\pi^{-1}(p) \subset \partial X$ or $\pi^{-1}(p) \subset X^{\circ}$. Thus we have a contradiction. Therefore $e=2$.

Lemma 2. Suppose $\partial X \neq \varnothing$. If $\pi$ is ramified above a boundary point of $D$, that is, $a_{k} \in \partial D$ for some $k$, then at least two of the fibers $\pi^{-1}\left(a_{i}\right) \subset \partial X$. Further the number of ramified fibers contained in $\partial X$ is even.

Proof. Suppose $a_{k} \in \partial D$ for some $k$.

If $\pi^{-1}\left(a_{k}\right) \subset \partial X$, then let $x \in \partial X$ such that $\pi(x)=a_{k} . \quad e_{\pi}(x)=2$ by Lemma 1 , and it is easy to see that there is an interior point $q \in X^{\circ}$ such that $\pi(q) \in \partial D$ (find charts as in the proof of Lemma 1 and look 
at $\xi=t \exp (\pi i / 2)$ for small enought $t)$. Thus regardless of whether $\pi^{-1}\left(a_{k}\right) \subset \partial X$ or $\pi^{-1}\left(a_{k}\right) \subset X^{\circ}$, there is an interior point $q \in X^{\circ}$ such that $\pi(q) \in \partial D$.

Now $\pi(\partial X)$ is a compact and hence closed subset of $\partial D$. Also, $\partial D \backslash \pi(\partial X) \neq \varnothing$. Topologically $\partial D$ is just a circle, of course. Therefore $\pi(\partial X)$ is a finite union of closed intervals.

It is easy to see that if $p$ is an end-point of one of these closed intervals, then $\pi$ is ramified above $p$ and $\pi^{-1}(p) \subset \partial X$. The number of such end-points is clearly even and not less than two.

Lemma 3. Suppose $X$ is orientable and $\partial X \neq \varnothing$. If $G \subset \operatorname{Aut}^{+}(X)$, then $\pi$ is ramified only above interior points of $D$.

Proof. Let $x \in X$, and consider the stablizer subgroup $S_{x}$ and its subgroup $S_{x}^{\prime}$. Since $G \subset \operatorname{Aut}^{+}(X), S_{x}=S_{x}^{\prime}$, directly from the definition of $S_{x}^{\prime}$. Consequently, if $x \in X^{\circ}$ then $\pi(x) \in D^{\circ}$ ( $\pi$ may or may not be ramified at $x$ ), and if $x \in \partial X$ then $e_{\pi}(x)=1$. Hence $\pi$ is ramified only above interior points of $D$.

C. $\gamma=0$, ramification above $\Phi^{\circ}$ only

Suppose $a_{1}, \cdots, a_{w} \in \Phi^{\circ}$ are interior points of $\Phi$. Then $n_{i}=2$ for each $i$, and by $(*)$

$$
\frac{2 g-2}{r}=-2+2 \sum_{i=1}^{\infty}\left(1-\frac{1}{k_{i}}\right)
$$

or

$$
\frac{g-1}{r}=\omega-1-\frac{1}{k_{1}}-\cdots-\frac{1}{k_{\omega}} .
$$

Again we see that $\omega \geqq 2$.

Suppose $\omega \geqq 3$. Since $k_{i} \geqq 2$ for each $i$, by (1)

$$
\frac{g-1}{r} \geqq \omega-1-\frac{\omega}{2} \geqq \frac{1}{2} \text {. }
$$

Hence $r \leqq 2(g-1)$.

Suppose $\omega=2$. $k_{1}=k_{2}=2$ is not a possibility, since that would imply $g=1$. Clearly then

$$
\frac{g-1}{r} \geqq 2-1-\frac{1}{2}-\frac{1}{3}=\frac{1}{6} .
$$

Hence $r \leqq 6(g-1)$.

These calculations have already yielded two interesting results:

THeOREM 3. Let $X$ be a compact Klein surface without boundary 
of genus $g \geqq 2$. If $G$ is a group of automorphisms of $X$ such that $X / G$ is the real projective plane $B$, then

$$
|G| \leqq 6(g-1) \text {. }
$$

Proof. $\partial B=\varnothing$, so the the theorem follows from calculations of $\S \mathrm{C}$.

THeORem 4. Let $X$ be a compact orientable Klein surface with boundary of genus $g \geqq 2$. Then

$$
\left|\operatorname{Aut}^{+}(X)\right| \leqq 6(g-1)
$$

and

$$
|\operatorname{Aut}(X)| \leqq 12(g-1)
$$

Proof. The first fact follows from the calculations of sections $\mathrm{A}$ and $\mathrm{C}$ and Lemma 3.

Either Aut $(X)=\operatorname{Aut}^{+}(X)$ or $\operatorname{Aut}^{+}(X)$ is a subgroup of Aut $(X)$ of index two. Thus the first fact implies the second.

D. $\gamma=0$, ramification above $\partial \Phi, \partial X \neq \varnothing$.

Now we assume that $X$ is a Klein surface with boundary. Then the quotient space $\Phi$ is the disc $\mathrm{D}$ (with its unique dianalytic structure).

We also assume that there is ramification above $\partial D$. By Lemma 2 , at least two of the fibers $\pi^{-1}\left(a_{i}\right) \subset \partial X$. We may suppose that this is the case for $a_{1}$ and $a_{2}$. Then $k_{1}=k_{2}=2$ by Lemma 1. $n_{1}=$ $n_{2}=1$, of course, so by $\left({ }^{*}\right)$

$$
\frac{2 g-2}{r}=-1+\sum_{i=3}^{\infty} n_{i}\left(1-\frac{1}{k_{i}}\right) .
$$

Therefore $\omega \geqq 3$ in this case.

First suppose $\omega \geqq 5$. Then by (2)

$$
\frac{2 g-2}{r} \geqq-1+(\omega-2) \cdot \frac{1}{2} \geqq \frac{1}{2} .
$$

Thus $r \leqq 4(g-1)$.

Next suppose $\omega=4$. There are three cases to consider, depending on whether there are 0,1 , or 2 of the points $a_{3}$ and $a_{4}$ on the boundary of $D$.

If $a_{3}, a_{4} \in D^{\circ}$, then

$$
\frac{2 g-2}{r} \geqq-1+2 \cdot \frac{1}{2}+2 \cdot \frac{1}{2}=1,
$$


and $r \leqq 2(g-1)$.

If one of the two points, say $a_{3}$, is a boundary point and $a_{4} \in$ $D^{\circ}$, then

$$
\frac{2 g-2}{r} \geqq-1+\frac{1}{2}+2 \cdot \frac{1}{2}=\frac{1}{2},
$$

and $r \leqq 4(g-1)$.

If $a_{3}, a_{4} \in \partial D$, then note that $k_{3}=k_{4}=2$ is not a possibility. Clearly then

$$
\frac{2 g-2}{r} \geqq-1+\frac{1}{2}+\frac{2}{3}=\frac{1}{6},
$$

and $r \leqq 12(g-1)$.

Finally, suppose $\omega=3$. Then from (2) we see that $n_{3}=2$, i.e., $a_{3} \in D^{\circ}$. Then

$$
\frac{2 g-2}{r}=1-\frac{2}{k_{3}} .
$$

Hence $k_{3} \geqq 3$ and $r \leqq 6(g-1)$ in this case.

A review of the calculations of $\S \S \mathrm{A}, \mathrm{C}$, and $\mathrm{D}$ gives our main result:

THeOREM 5. Suppose $X$ is a compact Klein surface with boundary of genus $g \geqq 2$. Then

$$
|\operatorname{Aut}(X)| \leqq 12(g-1) \text {. }
$$

4. Sharpness of the bounds. Here we consider three compact Klein surfaces of low genus and determine their automorphism groups directly.

EXAMPLE 1. Let $Y$ be a sphere with 3 holes, with the holes placed around the equator, centered around the vertices of an inscribed equilateral triangle. $Y$ is an orientable Klein surface of genus 2. $Y$ has a group (isomorphic to the dihedral group $D_{3}$ ) of orientationpreserving automorphisms of order 6 . Reflection in the plane of the equator is an orientation-reversing automorphism. $Y$ therefore has $12=12(2-1)$ automorphisms. The automorphism group is just $C_{2} \times D_{3}$, where $C_{2}$ denotes the cyclic group of order 2 .

ExAmple 2. Let $X$ be a sphere with 6 holes, with the holes centered around the vertices of an inscribed regular octahedron. $X$ is an orientable Klein surface of genus $5 . \quad X$ has a group of automorphisms isomorphic to the complete symmetry group (including 
reflections) of the regular octahedron, which is $C_{2} \times S_{4}$. Thus $X$ has $48=12(5-1)$ automorphisms.

EXAMPLE 3. Let $X$ be the Klein surface of Example 2, and let $\tau: X \rightarrow X$ denote the antipodal map. The quotient space $W=X / \tau$ is a real projective plane with 3 holes, a nonorientable Klein surface of genus 3. By considering the action of $C_{2} \times S_{4}$ on $X$, it is easy to see that there is a group of automorphisms of $W$ isomorphic to $S_{4}$.

Thus the bounds obtained in Theorems 4 and 5 are best possible. The bound $12(g-1)$ is attained for both orientable and nonorientable surfaces. Theorem 3 was obtained incidentally in our proof of Theorem 4. We do not know if the bound of Theorem 3 is the best possible.

In a forthcoming article [5] we study those finite groups that act as a group of $12(g-1)$ automorphisms of a compact Klein surface of genus $g \geqq 2$ with nonempty boundary. There we exhibit several infinite families of values of $g$ for which there is a compact Klein surface with boundary of genus $g$ that has $12(g-1)$ automorphisms.

5. Nevertheless it is possible to improve the bound $12(g-1)$ for a large number of topological types of Klein surfaces. Our main tool is a theorem of Maskit.

Let $X$ be a compact orientable Klein surface with boundary. By the analytic genus $p$ of $X$ we mean the topological genus of the compact surface $X^{*}$ obtained by attaching a disc to each boundary component of $X$. The relationship between $p$ and the (algebraic) genus $g$ of $X$ is given by

$$
g=2 p+k-1,
$$

where $k$ is the number of boundary components of $X$.

THEOREM 6. Let $X$ be a compact orientable Klein surface of genus $g$ with $k$ boundary components. If

$$
\frac{6(g-1)}{7}<k \leqq g-3 \text {, }
$$

then

$$
|\operatorname{Aut}(X)| \leqq 84(g-k-1)<12(g-1)
$$

Proof. Let $p$ be the analytic genus of $X$. Maskit has shown that there exists a compact Riemann surface $X^{*}$ of genus $p$ and an analytic embedding of $X$ into $X^{*}$ such that, under this embedding, every orientation-preserving automorphism of $X$ is the restriction of 
an orientation-preserving automorphism of $X^{*}$ [4, page 718]. Thus $\left|\operatorname{Aut}^{+}(X)\right| \leqq\left|\operatorname{Aut}^{+}\left(X^{*}\right)\right|$.

Now $2 p=g-k+1 \geqq 4$, so that $p \geqq 2$ and we may apply Hurwitz's bound for $\left|\operatorname{Aut}^{+}\left(X^{*}\right)\right|$. Hence $\mid$ Aut $(X) \mid \leqq 2 \cdot 84(p-1)=$ $84(g-k-1)$.

Note that $84(g-k-1)<12(g-1)$ if and only if $6(g-1)<7 k$.

If $g<16$, there are no integer values of $k$ such that $6(g-1) / 7<$ $k \leqq g-3$. The improved bound of Theorem 6 does apply to orientable Klein surfaces of genus 16 with 13 boundary components.

For large values of $g$ and suitable values of $k$, Theorem 6 gives a much better bound than Theorem 5. In fact, if $(g-k)$ is held fixed (that is, the analytic genus remains constant), Theorem 6 gives a uniform bound for the size of the automorphism group. On the other hand, there are orientable Klein surfaces with boundary of each genus $g \geqq 2$ to which Theorem 6 does not apply.

Finally, we obtain a similar result for nonorientable Klein surfaces with boundary.

THEOREM 7. Let $X$ be a compact nonorientable Klein surface of genus $g$ with $k$ boundary components. If

$$
\frac{6(g-1)}{7}<k \leqq g-2,
$$

then

$$
|\operatorname{Aut}(X)| \leqq 84(g-k-1)<12(g-1) .
$$

Proof. Let $\left(X_{0}, \nu, \tau\right)$ denote the orienting double of $X$, that is, $X_{0}$ is a compact orientable Klein surface with $2 k$ boundary components, $\nu: X_{0} \rightarrow X$ is an unramified 2-sheeted covering of $X$, and $\tau$ is the unique antianalytic involution of $X_{0}$ such that $\nu \circ \tau=\nu$. Further the genus $g^{\prime}$ of $X_{0}$ is $g^{\prime}=2 g-1$. For more details, see [1, pages 42-43].

Suppose $f: X \rightarrow X$ is an automorphism of $X$. Then there exists a unique orientation-preserving automorphism $\tilde{f}$ of $X_{0}$ such that

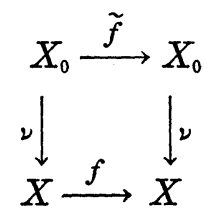

commutes $\left[1\right.$, page 42]. Hence $|\operatorname{Aut}(X)| \leqq\left|\operatorname{Aut}^{+}\left(X_{0}\right)\right|$.

Let $p^{\prime}$ be the analytic genus of $X_{0}$.

$$
p^{\prime}=\frac{(2 g-1)-2 k+1}{2}=g-k \geqq 2 .
$$


Then, using Maskit's theorem as in the proof of Theorem 6, we have that

$$
|\operatorname{Aut}(X)| \leqq\left|\operatorname{Aut}^{+}\left(X_{0}\right)\right| \leqq 84\left(p^{\prime}-1\right)=84(g-k-1) \text {. }
$$

As before, $84(g-k-1)<12(g-1)$ if and only if $6(g-1)<7 k$.

Note that the improved upper bound in Theorem 7 is the same as in Theorem 6 . The bound is applicable to a larger range of values of $g$ and $k$ in the nonorientable case, however.

The lowest genus to which Theorem 7 applies is the case of nonorientable Klein surfaces of genus 9 with 7 boundary components.

I am indebted to Robert Speiser not only for the suggestion that an approach similar to that of Hurwitz [2] might yield results about Klein surfaces but also for several helpful discussions. I would also like to thank Newcomb Greenleaf and Richard Preston for their advice. Finally, thanks are due the referee for bringing [4] to our attention and for making several helpful suggestions.

\section{REFERENCES}

1. N. Alling and N. Greenleaf, Foundations of the Theory of Klein Surfaces, Berlin: Springer-Verlag, 1971.

2. A. Hurwitz, Über algebraiche Gebilde mit eindentigen Transformationen in sich, Math. Ann., 41 (1879), 428-471.

3. I. Kra, Automorphic Forms and Kleinian Groups, Reading, Mass.: W. A. Benjamin, Inc., 1972.

4. B. Maskit. The conformal Group of a Plane Domain, Amer. J. Math., 90 (1968), 718-722.

5. C. May, Large Automorphism Groups of Compact Klein Surfaces with Boundary, (to appear).

6. D. Singerman, Automorphisms of Compact Non-orientable Riemann Surfaces, Glasgow Math J., 12 (1971), 50-59.

Received October 30, 1974 and in revised form February 6, 1975.

UNIVERSITY OF TEXAS 


\section{PACIFIC JOURNAL OF MATHEMATICS}

\section{EDITORS}

RICHARD ARENS (Managing Editor) University of California

Los Angeles, California 90024

\section{R. A. Beaumont}

University of Washington Seattle, Washington 98105
J. DugundJI Department of Mathematics University of Southern Californıa Los Angeles, California 90007

D. Gilbarg aNd J. Milgram Stanford University Stanford, California 94305

\section{ASSOCIATE EDITORS}

E. F. BeCKENBACH

B. H. NeUmanN

F. Wolf

K. YoSHIDA

\section{SUPPORTING INSTITUTIONS}

UNIVERSITY OF BRITISH COLUMBIA CALIFORNIA INSTITUTE OF TECHNOLOGY UNIVERSITY OF CALIFORNIA MONTANA STATE UNIVERSITY UNIVERSITY OF NEVADA NEW MEXICO STATE UNIVERSITY OREGON STATE UNIVERSITY UNIVERSITY OF OREGON OSAKA UNIVERSITY
UNIVERSITY OF SOUTHERN CALIFORNIA STANFORD UNIVERSITY UNIVERSITY OF TOKYO UNIVERSITY OF UTAH WASHINGTON STATE UNIVERSITY UNIVERSITY OF WASHINGTON AMERICAN MATHEMATICAL SOCIETY NAVAL WEAPONS CENTER 


\section{Pacific Journal of Mathematics \\ Vol. 59, No. 1 \\ May, 1975}

Shashi Prabha Arya and M. K. Singal, More sum theorems for topological

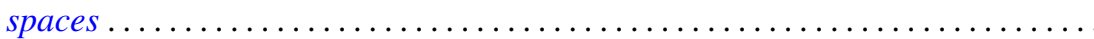

Goro Azumaya, F. Mbuntum and Kalathoor Varadarajan, On M-projective and

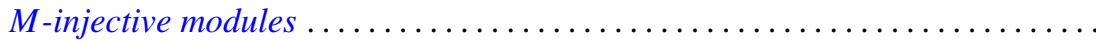

Kong Ming Chong, Spectral inequalities involving the infima and suprema of

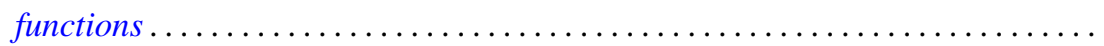

Alan Hetherington Durfee, The characteristic polynomial of the monodromy .......

Emilio Gagliardo and Clifford Alfons Kottman, Fixed points for orientation

preserving homeomorphisms of the plane which interchange two points ......

Raymond F. Gittings, Finite-to-one open maps of generalized metric spaces .......

Andrew M. W. Glass, W. Charles (Wilbur) Holland Jr. and Stephen H. McCleary,

$a^{*}$-closures of completely distributive lattice-ordered groups .............

Matthew Gould, Endomorphism and automorphism structure of direct squares of

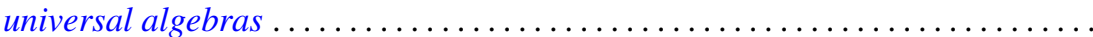

R. E. Harrell and Les Andrew Karlovitz, On tree structures in Banach spaces .....

Julien O. Hennefeld, Finding a maximal subalgebra on which the two Arens

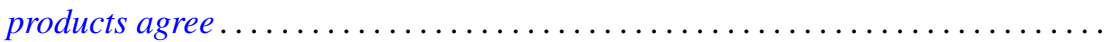

William Francis Keigher, Adjunctions and comonads in differential algebra .......

Robert Bernard Kelman, A Dirichlet-Jordan theorem for dual trigonometric

series

Allan Morton Krall, Stieltjes differential-boundary operators. III. Multivalued operators-linear relations...

Hui-Hsiung Kuo, On Gross differentiation on Banach spaces .

Tom Louton, A theorem on simultaneous observability ...

Kenneth Mandelberg, Amitsur cohomology for certain extensions of rings of algebraic integers.

Coy Lewis May, Automorphisms of compact Klein surfaces with boundary . . .

Peter A. McCoy, Generalized axisymmetric elliptic functions .

211

Muril Lynn Robertson, Concerning Siu's method for solving $y^{\prime}(t)=F(t$, $y(g(t)))$. .

Richard Lewis Roth, On restricting irreducible characters to normal

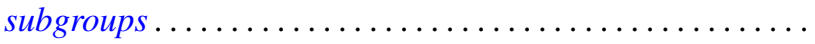

Albert Oscar Shar, $P$-primary decomposition of maps into an $H$-space .

Kenneth Barry Stolarsky, The sum of the distances to certain pointsets on the unit circle.

Bert Alan Taylor, Components of zero sets of analytic functions in $C^{2}$ in the unit ball or polydisc

Michel Valadier, Convex integrands on Souslin locally convex spaces ...

Januario Varela, Fields of automorphisms and derivations of $C$

Arnold Lewis Villone, A class of symmetric differential operators with deficiency

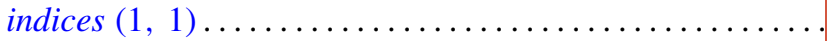

